\title{
SISTEMA COMUNAL, TRABAJO Y REPRODUCCIÓN AMPLIADA DE LA VIDA: HACIA FORMAS ALTERNATIVAS DE PRODUCIR Y VIVIR. UN ESTUDIO DE CASO EN SANTIAGO DEL ESTERO, ARGENTINA
}

COMMUNAL SYSTEM, WORK AND EXPANDED REPRODUCTION OF LIFE: TOWARDS ALTERNATIVE WAYS TO PRODUCE AND LIVE. A CASE STUDY IN SANTIAGO DEL ESTERO, ARGENTINA

SISTEMA COMUNAL, TRABALHO E REPRODUÇÃO EXPANDIDA DA VIDA: RUMO A FORMAS ALTERNATIVAS DE PRODUZIR E VIVER. UM ESTUDO DE CASO EM SANTIAGO DEL ESTERO, ARGENTINA

\section{María Victoria Suárez ${ }^{1}$}

\begin{abstract}
Resumen
El objetivo de este trabajo es analizar la complementariedad e integralidad entre los aspectos económicos, productivos y no económicos (particularmente sociales-afectivos) en Colonia Jaime, al identificar el proceso cotidiano que transforma lo individual en colectivo y en qué medida esta dinámica hace posible el desarrollo de las fuerzas productivas comunales sin romper su propia esencia. El estudio gira en torno a dos cuestiones centrales: 1) qué significa permanecer juntos para beneficiarse del trabajo común en Colonia Jaime y 2) cómo se manifiestan en el trabajo productivo cotidiano los procesos de apropiación colectiva de las herramientas y tecnologías que son por naturaleza individuales. Estas dos cuestiones entregan elementos conceptuales que permiten comprender cómo el desarrollo de las fuerzas productivas no implica necesariamente la disolución del sistema comunal, sino que por el
\end{abstract}

Doi: https://doi.org/10.15359/eys.24-56.5

Fecha de recepción: 17-09-2019. Fechas de reenvíos: 19-09-2019, 21-09-2019, 30-09-2019, 27-11-2019. Aceptado el 11-12-2019. Publicado el 11-12-2019.

${ }^{1}$ Dra. en Ciencias Sociales con Orientación en Geografía por la Universidad Nacional de Tucumán, Argentina. Becaria Posdoctoral del CONICET Temas Estratégicos. Integrante del Equipo de Sociología Rural del Instituto de Estudios para el Desarrollo Social (INDES/FHCSyS/UNSE-CONICET), Santiago del Estero, Argentina. Correo electrónico: victoriasuarez_4@hotmail.com ORCID https://orcid.org/0000-0001-9669-4825 
contrario lo potencia y reafirma, aunque siempre reconoce la presencia de contradicciones, tensiones y conflictos al interior de la propia comunidad. Colonia Jaime muestra otra forma de producir alimento y de vivir, que escapa al ordenamiento asignado por la lógica capitalista. Los ejemplos extraídos muestran que la teoría del valor es pensada desde la coproducción y colaboración entre ser humano y naturaleza y entre ellos mismos. La estrategia metodológica reúne tres aspectos principales: análisis de fuentes documentales; entrevistas y observación participante.

Palabras claves: economía para la vida; fuerzas productivas; sistema comunal

\begin{abstract}
The objective of this work is to analyze the complementarity and integrality between the economic, productive and non-economic aspects (particularly social-emotional) in Colonia Jaime, by identifying the daily process that transforms the individual into a collective and to what extent this dynamic makes possible the development of communal productive forces without breaking their own essence. The study revolves around two central issues: 1) what it means to stay together to benefit from common work in Colonia Jaime and 2) how the processes of collective appropriation of tools and technologies that are by nature individual are manifested in daily productive work. These two issues provide conceptual elements that allow us to understand how the development of the productive forces does not necessarily imply the dissolution of the communal system, but instead strengthens and reaffirms it, although it always recognizes the presence of contradictions, tensions and conflicts within the own community. Colonia Jaime shows another way of producing food and living, which escapes the system assigned by capitalist logic. The examples drawn show that the theory of value is thought from the co-production and collaboration between human beings and nature and among themselves. The methodological strategy has three main aspects: analysis of documentary sources; Interviews and participant observation.
\end{abstract}

Keywords: economy for life; productive forces; communal system 


\begin{abstract}
Resumo
O objetivo deste trabalho é analisar a complementaridade e a integralidade entre os aspectos econômicos, produtivos e não econômicos (particularmente socioemocionais) de Colonia Jaime, identificando o processo cotidiano que transforma o indivíduo em coletivo e em que medida essa dinâmica possibilita a desenvolvimento de forças produtivas comunitárias sem quebrar sua própria essência. 0 estudo gira em torno de duas questões centrais: 1) o que significa permanecer juntos para se beneficiar do trabalho comum em Colonia Jaime e 2) como os processos de apropriação coletiva de ferramentas e tecnologias que por natureza são individuais se manifestam no trabalho produtivo diário. Essas duas questões fornecem elementos conceituais que nos permitem entender como o desenvolvimento das forças produtivas não implica necessariamente a dissolução do sistema comunal, mas o fortalece e reafirma, embora reconheça sempre a presença de contradições, tensões e conflitos dentro do sistema. própria comunidade. Colonia Jaime mostra outra maneira de produzir comida e viver, que escapa ao sistema atribuído pela lógica capitalista. Os exemplos apresentados mostram que a teoria do valor é pensada a partir da coprodução e colaboração entre seres humanos e natureza e entre si. A estratégia metodológica possui três aspectos principais: análise de fontes documentais; Entrevistas e observação participante.
\end{abstract}

Palavras-chaves: economia para a vida; sistema comunitário; forças produtivas

\title{
Introducción
}

Pensar los sistemas comunales como formas de organización de la vida social, capaces de resistir y adaptarse al contexto capitalista, conduce a poner en tensión los postulados clásicos tanto liberales como marxistas, según los cuales el comunalismo representa un obstáculo al desarrollo de las fuerzas productivas. Asimismo, supone asumir una posición crítica hacia "uno de los postulados centrales del capitalismo que plantea el lucro y la ganancia como principales motores del crecimiento y desarrollo de las fuerzas productivas" (Suárez y Paz, 2018, p. 265). Asociado fuertemente a la existencia de la propiedad privada individual de los medios de producción como de la mercancía que de ellos se deriva.

Precisamente, ante los efectos devastadores de un capitalismo atroz, numerosas voces se levantaron tanto desde los ámbitos académicos, como de los movimientos sociales y los espacios 
de militancia en busca de experiencias donde las relaciones capitalistas no sean el principio organizador en la vida social de los sujetos involucrados. Efecto de esto, existe alguna evidencia a partir de estudios de caso que destacan los esfuerzos que vienen realizando distintas comunidades y actores rurales por construir modelos económicos, sociales y políticos alternativos donde sean posibles los procesos de reproducción sostenidos y muchas veces ampliados (Gutiérrez y González, 2016; Gracia, 2015; Santos, 2011; Paz y de Dios, 2011; Van der Ploeg y Mardsen, 2008; Patzi, 2004).

Esto significa poner en discusión la posibilidad de ciertas instancias de "acumulación nocapitalista", entendida como el "proceso social de transformación del excedente económico obtenido tendiente a lograr la reposición y ampliación de sus medios de producción como la satisfacción de las necesidades" de los comuneros (Barkin y Rosas, 2006, p. 2). Por lo tanto, este tipo de acumulación no-capitalista se apoya sobre estrategias alternativas de producción, distribución y comercialización que elevan el nivel de vida de las comunidades en un marco de protección del medio ambiente, la ayuda mutua y la acción colectiva como forma de vida.

En palabras de Hinkelammert y Mora (2005) las acciones están orientadas por "una racionalidad más integral de respeto al circuito natural de la vida humana" que prioriza un "sujeto vivo, corporal, concreto, necesitado" y su relación con "el arte de gestionar los bienes necesarios para abastecer la comunidad y satisfacer las necesidades humanas (oikonomía)" (p. 19). Por lo tanto, la vigencia y resiliencia de estas formas de vida frente a los cambios en el contexto donde se insertan pone en evidencia que la incorporación de ciertas prácticas capitalistas, específicamente del dinero como modalidad de intercambio y el vínculo con ciertos mercados e instituciones públicas y privadas, no significaron la desaparición de la comunidad (Osorio, 2013; Ruiz, 2012; Giménez, 1991).

El propósito de este artículo está dirigido a comprender algunas de las estrategias al interior de un sistema comunal que potenciaron el desarrollo de las fuerzas productivas permitiendo construir caminos alternativos en un contexto capitalista, donde la producción toma como prioridad una economía para la vida (Hinkelammert y Mora, 2005). El caso está representado por Colonia Jaime, una comunidad agropecuaria que se ubica en el área de riego de la provincia de Santiago del Estero, Argentina. Colonia Jaime se reconoce como una institución de naturaleza filosófica (Asociación Civil Colonia Jaime), sin fines de lucro y de bien común, fundada sobre los principios doctrinarios-filosóficos de la Escuela Magnético Espiritual de la Comuna Universal (E.M.E de la C.U) ${ }^{2}$. Está asentada sobre un predio de cerca de 600 hectáreas de las cuales 350 están ocupadas con actividades productivas y la casa comunal. Fue fundada en el año 1932 por un filósofo español llamado Joaquín Trincado, quien organizó la comunidad a partir de ciertos

\footnotetext{
${ }^{2}$ Para más información acerca de los fines que persigue la comunidad consultar el sitio oficial de la comunidad: http://www.coloniajaime.org/
} 
principios éticos que regularían la vida tanto en sus aspectos sociales, como económicos, productivos y educativos. Está conformada por cerca de 85 personas que se distribuyen en unas 25 familias. Hacia el interior de la comunidad no existe la propiedad privada individual y el ámbito privado de las familias se reserva a las habitaciones que están equipadas con todo lo necesario para aliviar las actividades domésticas. Mientras que comedor, cocina, e instalaciones productivas, de esparcimiento y educativas son comunales. Las actividades que realizan son muy variadas, ya que buscan autoabastecer a la comunidad con su propia producción. Sin embargo, los productos que se obtienen de las distintas actividades agrícolas, pecuarias y elaboradas a partir de agregado de valor compiten en los mercados con empresas capitalistas: la producción de leche bovina, de lechones, chacinados, verduras y hortalizas de estación, miel de abejas, pollos, milanesas de carne de pollo, entre otros. También han desarrollado un sistema de venta directa en la ciudad, mediante dos locales (granjas urbanas) donde venden todos sus productos y revenden otros que no los generan dentro de la comunidad.

La temática propuesta será abordada a partir de dos frases que reflejan la lógica que subyace en Colonia Jaime y expresan los lineamientos organizativos dejados por su fundador. La primera es "El fin del progreso es el mayor bienestar de la comunidad" (Trincado, 1975 [1934]) y expresa un interés por permanecer juntos para beneficiarse no solo del trabajo comunal en el proceso de producción sino también beneficiarse a partir del trabajo solidario y compartido entre los propios comuneros, en los momentos que ese sujeto vivo y necesitado de las condiciones materiales, espirituales y sociales así lo requiera (Meillassoux, 1987; Hinkelammert y Mora, 2005). Desde este enunciado entonces, se buscará indagar los mecanismos que se disparan en la Colonia cuando las necesidades privadas de algunos de sus integrantes se vuelven comunes y son resueltas comunalmente. La otra proposición es "El trabajo productivo regenera al hombre y da derecho al consumo" (Trincado, 1983 [1922], p. 80). En esta línea la creación de valor para generar procesos de acumulación es central, en términos de cubrir las necesidades de los comuneros. Estos fueron posibles a partir del desarrollo de las fuerzas productivas, en ausencia del salario y la propiedad privada individual, con importantes incorporaciones tecnológicas, que se orientan tanto a la producción como a su vida cotidiana. En ese marco, se examinará la relación del trabajador comunero con los medios de producción y con la comunidad, como aspectos esenciales para comprender las estrategias por las cuales el desarrollo de las fuerzas productivas no resquebrajó ni cristalizó la potencialidad comunal.

Esta investigación se enmarca en una más amplia que culminó con la tesis doctoral titulada "Estrategias productivas y ética espiritual en la gestión de los recursos comunes en Colonia Jaime (Santiago del Estero, Argentina)" (Suárez, 2019) ${ }^{3}$. La estrategia metodológica incluyó análisis de fuentes documentales; entrevistas en profundidad y observación participante. El trabajo de

\footnotetext{
${ }^{3}$ La tesis doctoral fue defendida en marzo de 2019, con lo cual se obtuvo el título de Doctora en Ciencias Sociales (orientación Geografía) en la Facultad de Filosofía y Letras de la Universidad Nacional de Tucumán.
} 
campo se realizó a partir de estadías cortas, pero con cierta periodicidad en la comunidad entre los años 2014-2017. Las fuentes documentales que se trabajaron, en este caso, fueron algunos de los libros que componen la obra escrita del Espiritismo Luz y Verdad con autoría de Joaquín Trincado, específicamente, Código de Amor Universal Tomo I y Tomo II. Además, el estatuto de la Escuela Magnético Espiritual de la Comuna Universal; el reglamento interno de Colonia Jaime del año 1932 y el actual estatuto de la comunidad. Las entrevistas fueron realizadas a los comuneros encargados de las distintas actividades productivas, administrativas, educativas y recreativas; a la actual presidente de la comunidad y a dos matrimonios jóvenes que efectuaron su alianza durante el período de la investigación. Durante el trabajo de campo la observación participante como técnica para la recolectar la información resultó fundamental, en tanto se pudo registrar percepciones, situaciones y hechos históricos a partir de encuentros espontáneos con los comuneros, en distintos espacios, dentro y fuera de la comunidad.

\section{La centralidad del trabajo y la potencialidad de la cooperación más allá de lo económico.}

Colonia Jaime fue fundada por el español Joaquín Trincado ${ }^{4}$, quien dejó establecido un modo de organización social, económica y educativa. Toda comunidad que se constituyera bajo sus principios debía cumplir con los fines establecidos, con la forma organizativa comunal y procurar el bienestar de sus integrantes. El primer reglamento manifestaba al respecto que "la Colonia atiende y cubre todas las necesidades materiales de los comuneros en alimentos, vestidos, asistencia médica, instrucción escolar y vivienda" (Reglamento Interno de Colonia Jaime, 1931, p. 5). Para esto,

La economía impone que cada hombre y mujer de la Colonia, considere suyo todo para su cuidado, mejoramiento y acrecentamiento de los intereses comunales, por lo cual cooperarán [...] no sólo para bastarse la Colonia a sí misma sino para adquirir capitales por la venta de productos agrícolas y de granja, bajo el lema de, todos para todos y nadie individual. (Reglamento Interno de Colonia Jaime, 1931, p. 6)

Por lo tanto, los intereses y preocupaciones que corresponden al ámbito privado de cualquier persona son resueltos comunalmente por la institución.

El eje central que sostiene este sistema es el trabajo que posiciona al hombre como principal motor y artífice de la vida comunal. En esta línea de pensamiento el hombre no aparece aquí separado de los medios materiales de trabajo como tampoco de la propia Naturaleza, lo que busca una relación de complementación y sinergia (Paz, 2017). Esta perspectiva, a diferencia de

\footnotetext{
${ }^{4}$ Para conocer algunos de los lineamientos sobre los cuales se organiza Colonia Jaime y que fueron establecidos por su fundador, consultar el trabajo de Suárez y Paz (2017).
} 
la lógica capitalista donde la interacción del hombre, los medios de producción y la Naturaleza son atraídos por la órbita del mercado, muestra un sistema comunal donde la intencionalidad última de la producción no es la ganancia, sino tiene como principio central la subsistencia y la satisfacción integral de las personas que conforman la comunidad.

Si bien en Colonia Jaime, el trabajo está orientado a la generación de valor a partir de la producción de bienes de consumo y de mercancía (como se verá en el próximo punto), buena parte del trabajo tiene como finalidad la ayuda mutua, el compromiso y la contención social del grupo de comuneros. Para ello es imprescindible una condición técnico-productiva y medios de producción que estén en concordancia y simetría con las fuerzas laborales mínimas necesarias que le permita, con palabras de García (2009) la conformación de una "célula productiva" básica y, "en torno a ella, la forma social equivalente de la reproductividad social" (p. 273).

El trabajo colectivo se expresa así en su doble carácter de obligación y posibilidad. "En su aspecto obligatorio, el trabajo crea, se sostiene en el tiempo y consigue mediante esta dinámica, fortalecer los lazos sociales, crear vínculos y afrontar desafíos comunes" (Flores et al., 2014, p. 1649), buscando conservar las condiciones que aseguren la permanencia colectiva. En tanto posibilidad, el trabajo manifiesta la oportunidad permanente de alcanzar la aceptación, de ser reconocido como comunero en la actividad laboral cotidiana (Osorio, 2013; Martínez, 2003). "EI trabajo comunitario tiene que ver con el compromiso", sin que se transforme en una relación mercantilizada, sino que esté orientado por "otro tipo de valoraciones como el desarrollo de prestigio local o de compromisos comunales" (Flores et al., 2014, p. 1649).

El trabajo en Colonia Jaime se manifiesta en distintas prácticas, desde las productivas hasta las actividades doméstico-cotidianas como el cuidado de los niños, la asistencia a las nuevas familias comuneras, acompañamiento y cuidado de los ancianos, entre otras. Esto implica una conciencia moral acerca de las obligaciones, los derechos y los deberes con y para la vida en común que busca la igualdad de las personas y de oportunidades. Es así que todas las actividades comunales requieren de un acuerdo voluntario de adscripción de los comuneros, a partir del cual, ninguno queda desprovisto de los medios materiales de vida y trabajo individuales y colectivos o de la "capacidad de obtenerlos rápidamente, y de su vinculación libre con la tierra que se presenta socialmente y existe técnicamente como patrimonio común de todos" y de nadie en particular (García, 2009, p. 256).

Cuando un comunero decide formar una familia y constituirse como matrimonio se activan dispositivos normativos de consulta, organización, compromiso y aceptación de las normas preestablecidas. Un caso interesante fue el embarazo de una joven comunera que cursaba sus estudios universitarios en la ciudad capital de Santiago del Estero. Ante la situación, toda la comunidad se despliega y el Consejo convoca a reunión a la joven pareja para consensuar acuerdos respecto a la formalización de la unión conyugal ante las leyes civiles y la autoridad 
comunal $^{5}$ (en tanto norma de convivencia necesaria en la Colonia); la organización de la fiesta de casamiento y el viaje de luna de miel ${ }^{6}$ (una tradición muy respetada por la comunidad); la asignación del espacio y construcción de un departamento ${ }^{7}$ de aproximadamente 50 metros. cuadrados, como único espacio privado para la reproducción de la familia; así como la liberación de las tareas comunales de la mujer durante el embarazo. Finalmente se da inicio a un período de prueba para el joven que vive fuera de la comunidad. Este período consiste en la participación en las distintas actividades o rubros que allí se realizan para conocer las tareas que implica y sobre todo de las sesiones de estudio como espacio de formación, discusión y apropiación de los principios filosóficos fundantes.

En la primera reunión avisamos del embarazo y la decisión de casarnos, en la siguiente hablamos de la fiesta cómo queríamos que sea, chica, grande, en qué fecha. Todo dependía cuando terminaba de trabajar yo, me quedaban dos o tres meses más. La idea era hacer algo sencillo con cien invitados con los más cercanos porque hace dos meses ya se había organizado un casamiento, pero todos nos han apoyado y ha terminado siendo más grande como se acostumbra aquí. (entrevista a comunero, 13 de junio de 2017)

Yo estaba estudiando y también se ha hablado en la reunión sobre mi situación, qué iba a hacer yo, si iba a seguir estudiando o no, yo he elegido dejar por ese tiempo porque para mí entre estar embarazada, el casamiento y tantas cosas de golpe, prefería vivir el embarazo tranquila y no con tantas cosas que por ahí me hagan mal, de querer exigirme, así que digo no, lo vivo tranquila. (entrevista a comunera, 13 de junio de 2017)

Se puede observar en estos fragmentos una economía orientada hacia la vida (Hinkelammert y Mora, 2003) donde la reproducción de la vida humana no está atravesada por la mercantilización. Es decir que, mientras en la sociedad cada individuo debe disponer de un fondo de dinero para poder resolver necesidades fundamentales en materia de salud, vivienda, alimentación,

\footnotetext{
${ }^{5}$ Durante el acto civil de matrimonio se firman dos actas, una que da cumplimiento a las leyes civiles de la provincia de Santiago del Estero y otra que los reconoce como matrimonio ante la Escuela Magnético Espiritual de la Comuna Universal con la identificación de "unión de hermanos". De esta forma quedan reconocidos como matrimonio ante ambas autoridades.

${ }^{6}$ Puesto que uno de los integrantes de la pareja no era miembro de la comunidad, recibieron aportes económicos de familiares externos para la realización del evento y el equipamiento del departamento dentro de la Colonia. Asimismo, el joven accedió a un préstamo personal que le fue otorgado en su trabajo para colaborar con los gastos, lo cual fue aceptado por la comunidad con la condición de acabar sus compromisos económicos una vez asentado en ella.

7 Los comuneros acostumbran denominar a este espacio "habitaciones", las cuales se encuentran ubicadas consecutivamente conformando algo así como pabellones enfrentados mediados por un jardín.
} 
educación y crianza de los niños, en el espacio comunal de Colonia Jaime es la institución misma la que vela por todos sus integrantes. La vida de cada uno de ellos es responsabilidad de la comuna, pero al mismo tiempo cada comunero es responsable de la reproducción de la comunidad. Esta relación individuo-comunidad se crea, recrea y reproduce a partir del trabajo con el que se producen los medios para la vida en común.

La joven pareja tuvo su fiesta de casamiento y la luna de miel luego de haber superado el período de prueba. Colaboraron en la construcción del departamento hasta el día del viaje, tuvieron libertad para la elección del decorado interno, más allá de la estructura edilicia preestablecida por norma comunal (dos habitaciones, una matrimonial y otra para los hijos, un baño y un pequeño espacio para electrodomésticos como lavarropas). Durante su viaje fueron los comuneros quienes finalizaron el trabajo y en su regreso todo estaba listo.

Para cuando se instalaron en la comunidad, el joven esposo ya había renunciado a su trabajo en la ciudad y a todos sus bienes materiales llevando consigo únicamente algunas prendas de vestir para incorporarse en forma definitiva a las tareas de la huerta, rubro escogido para desplegar sus habilidades como trabajador-comunero. En tanto que su estadía definitiva en la Colonia fue aprobada por la Asamblea General de Miembros tal como lo establece el Estatuto.

Lo que preocupaba aquí era mi trabajo, si yo qué iba a ser, iba a dejar o no iba a dejar. Mi jefe me había ofrecido un departamento a la vuelta del trabajo, pero ya habíamos tomado la decisión de vivir aquí. El auto le he dejado a mi mami y me decían que estaba loco. La gente no entendía que llegue fin de mes y yo no maneje plata. Para ellos pasa más por lo material que por el bienestar de uno. (entrevista a comunero, 13 de junio de 2017)

Esta preocupación tiene que ver con el hecho de que todos los comuneros realizan trabajos en y para la comunidad, sin ningún tipo de retribución en forma de salario, lo que no significa que algún de ellos no tenga la posibilidad de tener su empleo fuera de esta y destinar los recursos que adquiera totalmente a la comunidad, pero esto no es muy aplicable porque no está previsto en su forma organizativa. $Y$ aquel joven decidió renunciar a su vida externa para abocarse a la comuna.

Es en este momento cuando se firma, simbólicamente, el contrato social colectivo que le asigna una función en la comunidad y a partir del cual se dirige por el interés del todo. Como dice Luis Villoro (2003) es a partir de este contrato que:

Cada individuo se considera a sí mismo un elemento perteneciente a una totalidad, de manera que lo que afecta a ésta le afecta a él: al buscar su propio bien busca el del todo. En toda comunidad existe una tensión entre los intereses particulares y 
los del todo. Sólo cuando los sujetos de la comunidad incluyen en sus deseos lo deseable para todos, la comunidad se realiza cabalmente; cuando no es así, permanece como una meta regulativa, a la que podemos acercarnos sin alcanzarla. (pp. 48-49)

Existen entonces, entre los comuneros "intereses de permanecer juntos para beneficiarse de su trabajo común" (García, 2009, p. 272), de manera que el progreso tenga como fin el mayor bienestar de la comunidad. Allí la cooperación aparece como forma organizativa en la que la fuerza de trabajo individual se manifiesta como fuerza de trabajo social colectiva, dirigida a un fin específico y la modalidad técnica en la que ella se presenta como condición de trabajo en el proceso de trabajo: "Será entonces el núcleo en torno al cual girará el conjunto de funciones sociales circulatorias, rituales y simbólicas que caracterizan a las entidades comunales y definen la específica forma social en la que los miembros de la comunidad quedan fusionados vívidamente" (García, 2009, p. 274).

\section{Sobre la relación trabajador-medios de producción-comunidad y las formas de apropiación colectiva.}

(...) nuestro régimen Comunal -no "comunista"- que en síntesis es: trabajar todos para todos en verdadera fraternidad, procurando que la Madre Naturaleza nos dé el mayor rendimiento posible para invertir todos sus productos en extender cada vez más los cultivos y hacer cada vez más grata, material y espiritualmente, la vida de los que trabajan sin privilegios ni excepciones, porque entendemos que el "trabajo productivo" es sólo lo que regenera a los hombres y los prepara en aquellas condiciones para deponer antagonismos y gozar de la verdadera fraternidad. Esos son en resumen los fines prácticos que persigue esta institución (...). (Trincado, 15 de abril de 1935, pp. 12-13)

Marx (1987) consideraba que en las formas económicas precapitalistas el trabajo comunal no estaba orientado a la creación de valor, sino que tenía como fin primordial la conservación de la propiedad y de toda la comunidad. A diferencia de esta afirmación, en Colonia Jaime el trabajo responde a un doble propósito: la creación de valor a través de la producción de bienes de consumo y de mercancía sin que exista producción de plusvalía y a través de este proceso, a la reproducción misma de la comunidad. Esta creación de valor le permitió generar una instancia de acumulación que se sustenta sobre una valoración del trabajador colectivo por sobre los medios de trabajo, pero también se basa en la ausencia del salario, en una fuerza productiva que se orienta tanto a los valores de uso como a los de cambio y en un cierto nivel de dominio de la naturaleza por parte de los comuneros. En este sentido, "para que la apropiación real del proceso de trabajo comunal no sea baja e instale desde el principio un desarrollo limitado que seguramente la llevará a la ruina, se requiere el aumento de la productividad del trabajo que se logra a partir del desarrollo de las fuerzas productivas" (Suárez y Paz, 2017, p. 105). 
A este respecto, si bien la primera gran fuerza productiva es la comunidad misma, el desarrollo tecnológico aparece como un elemento fundamental para pasar de una economía de subsistencia a una de mayor escala, con un ingreso económico comunal que le permita acumular. En la experiencia de Colonia Jaime, la construcción de redes de apoyo mutuo con distintas instituciones estatales facilitó el asesoramiento y apoyo financiero para introducir ciertas innovaciones tecnológicas (en producción primaria y de industrialización de los productos primarios) que se adecuaban a sus objetivos, aspiraciones y posibilidades como comunidad ${ }^{8}$. Colonia Jaime pudo apropiarse del entorno liberal sin que esto implique la transformación del sistema de vida. La clave está en el tipo de interconexión entre el trabajador colectivo y las herramientas o medios de trabajo, donde el primero no está subordinado a la herramienta como sucede en el régimen capitalista, sino que su función es hacer efectiva y potenciar la destreza, inteligencia y energía del comunero, al buscar el progreso para el mayor bienestar de la comunidad. Es decir que, existe "una asociación directa entre la utilidad del medio de trabajo y la destreza individual" que "no es de especialización exclusiva en una rama de la laboriosidad, sino que es pericia socializada y desarrollada por todos los trabajadores como un todo, como parte de un conocimiento general extendido" (García, 2009, pp. 275-276). Entonces, a diferencia de lo que ocurre en el la forma capitalista de producción, en un sistema comunal se generan relaciones de armonía y colaboración entre los medios de trabajo y los comuneros.

Una de las tecnologías que fue apropiada por la comunidad es el Biodigestor. Esta tecnología les permitió transformar las excreciones de los animales (principalmente vacunos) para la producción de gas, que es utilizado como combustible en la cocina comunal. Actualmente la comunidad está proyectando su ampliación de modo que el biogás alimente también a la sala de elaboración de chacinados y la sala de faena de pollos. Por otro lado, el biodigestor impactó en forma directa el sistema agroecológico al minimizar el consumo de leña; evitar la degradación del monte; disminuir la contaminación de los vectores que afectan a personas y animales y al reutilizar el agua tratada en el riego de las parcelas en producción ${ }^{9}$. Los comuneros lo califican como una herramienta de "muy alta tecnología" por estar automatizado: "los bastidores y el compresor arrancan solos, los bioreactores mantienen la temperatura gracias a una circulación de agua permanente que también es automática y se necesita una sola persona para hacer la carga, lo demás se maneja sólo" (entrevista a comunero, 14 de junio de 2017).

Esta tecnología como medio de producción, es por naturaleza individual, solo a partir del trabajo colectivo y en el proceso de apropiación del producto resultante de la interconexión trabajadorherramienta es que se transforma en comunal. Pues el biodigestor no demanda demasiada mano

\footnotetext{
${ }^{8}$ En Suárez y Paz (2017), existe un desarrollo exhaustivo sobre la relación de la Colonia Jaime con el Estado, en especial, en lo que tiene que ver con la innovación tecnológica.

${ }^{9}$ El proyecto fue desarrollado en el año 2011 por el INTI (Instituto Nacional de Tecnología Industrial), con la participación de la Universidad Nacional de Santiago del Estero y fue financiado por la Subsecretaría de Desarrollo, Ciencia y Tecnología y Gestión Pública del Gobierno de la provincia de Santiago del Estero con fondos propios.
} 
de obra para su ejecución, sino más bien, tareas de mantenimiento, control y reparación lo que demanda la atención de solo dos comuneros, que al mismo tiempo realizan otras actividades. Es el producto obtenido (biogás y abono líquido) lo que se convierte en un servicio común pues beneficia a todos los comuneros al asegurar la cocción de los alimentos de cada día y la fertilización de la tierra para la agricultura.

Otro ejemplo de equipamiento para la producción que es individual, pero requiere coordinación de tareas y genera bienes de consumo de aprovechamiento comunal y bienes de cambio para obtener dinero, es la sala de elaboración de chacinados. Al mismo tiempo que potencia la mano de obra y mejora el proceso productivo, logra mayor eficiencia energética, material y financiera, incrementa el valor agregado y el valor comercial, a partir de su calidad, manifiesta en la fuerte demanda de los consumidores por los clásicos ${ }^{10}$ chorizos de la Colonia. En los 20 años que Colonia Jaime lleva vendiendo sus productos en las granjas de la ciudad de la Banda, los comuneros trabajaron cuidadosamente para garantizar la sostenibilidad de la producción y evitar excedentes de producción. En este sentido, llevar un control de las ventas; entonces, si bien no pudieron consolidar (hasta ahora) una gestión de todo el sistema de producción, el conocimiento obtenido "a partir de los trabajos con la naturaleza y los vínculos con los clientes y proveedores de algunos productos, constituye el recurso de mayor importancia que conecta todos los eslabones de una larga cadena de producción, circulación y consumo" (Rodríguez et al., 2015, p. 14). La construcción de dicha fábrica de chacinados pudo ser posible a partir de un subsidio otorgado por el estado provincial (para la compra de la cámara frigorífica), mientras que el Ministerio de la Producción de la provincia brindó asesoramiento técnico durante el proceso de ejecución y los comuneros fueron quienes construyeron el espacio de producción con sus propios medios económicos y materiales, como de mano de obra (Suárez y Paz, 2017).

Finalmente, la sala de faena de pollos es otro ejemplo que visibiliza la incorporación de medios de trabajo individuales, que no fueron obtenidos con la colaboración de instituciones públicas, sino más bien fruto de las relaciones sociales que construyen, en este caso, donadas por simpatizantes del modelo de vida comunal y ampliados por recursos propios de la comunidad. Día tras día se activa en este espacio de producción una interconexión entre la capacidad de trabajo y la herramienta de trabajo. Ambos elementos se complementan para generar un producto fresco para el consumo interno y su comercialización, de lo cual se deriva un sentido o una percepción social del consumidor muy importante que lo diferencia de una economía

\footnotetext{
${ }^{10}$ La producción de chacinados se realiza en la comunidad desde su fundación, aunque con fines de autoconsumo hasta que se comenzaron a comercializar entrada la década de 1990. Fueron los primeros habitantes (descendientes de inmigrantes italianos, yugoslavos, españoles y algunas familias santiagueñas) quienes fabricaron los primeros chorizos italianos y criollos, a partir de recetas tradicionales que se transmitieron de generación en generación (Rodríguez, et al., 2015).
} 
capitalista: para Colonia Jaime el consumidor no representa un numero sino un ser vivo que debe ser cuidado del mismo modo que la comunidad cuida de sus comuneros.

Así, el hecho de que en Colonia Jaime, la incorporación de tecnología en todas sus formas para la ampliación y mejoramiento de sus actividades productivas haya permitido procesos de acumulación no-capitalista y un desarrollo de las fuerzas productivas sin comprometer el modelo de vida comunal, tiene que ver con que las herramientas permiten "el flujo efectivo de la energía e inteligencia viva" del trabajador y estas "existen como las principales y mayoritarias fuerzas productivas" de la comunidad (García, 2009, p. 275); sin embargo, tales herramientas no subordinan el trabajo de los comuneros que se manifiesta como un saber hacer, una artesanía y una instancia creativa y no alienadora.

Mucho ha costado llevar adelante este principio de vida comunal, este deseo, de que los que vivimos aquí en Colonia Jaime, sentimos que estamos aquí voluntariamente para cumplir un fin social, para trabajar en equipo, para compartir, para interactuar y eso es lo que venimos haciendo en todos estos años. (Diario Panorama, 2012)

Entonces cuando Trincado dice que el trabajo productivo regenera al hombre y da derecho al consumo está queriendo significar que el trabajo humano colectivo no tiene como fin el lucro o la ganancia sino más bien generar los medios materiales de vida necesarios para la reproducción, alcanzados a partir del consumo que pertenece como tal, a un orden ético o moral de la vida antes que al orden específicamente económico. Es decir, en la medida que cada comunero trabaje por y para el todo, sus necesidades serán satisfechas por derecho, hay por tanto una relación dialéctica trabajo-consumo y trabajador-comunidad.

\section{A modo de cierre}

En el espacio rural existen diferentes formas de organización social que dan lugar a distintos procesos colectivos que desembocan, muchas veces, en cooperativas; sociedades de fomento rural; colonias, encierros ganaderos comunitarios, reservas campesinas entre otros; los cuales se organizan ya sea por tipo de producción, para acceder a capacitación o asistencia técnica, o en torno a problemáticas sociales y defensa gremial o incluso con la intención manifiesta de servir como ejemplo de organización comunal en oposición a las formas capitalistas y liberales.

Colonia Jaime surge como una colonia comunal que se organiza alrededor de una doctrina filosófica que discute los modos de vida impuestos por el capitalismo, que alrededor de los años 30 ya comenzaba a mostrar ciertas debilidades. La forma comunal constituía para su fundador y seguidores la forma de organización social que permitiría al hombre desplegar su potencial como ser humano y espiritual en un marco de igualdad, solidaridad, respeto por la naturaleza y trabajo 
colectivo. Colonia Jaime lleva más de 80 años conservando esos principios fundantes, aunque en diálogo con la sociedad capitalista que la rodea incorporando ciertas prácticas, pero subordinándolas a la lógica comunal. En este sentido, recuperar la experiencia de Colonia Jaime permite pensar al sistema comunal como un espacio de integralidad y complementariedad entre dinámicas económicas y no económicas (culturales, sociales, afectivas, políticas) capaz de sostenerse en el tiempo por la voluntad cotidiana manifestada en las prácticas y decisiones de los actores involucrados.

A diferencia del sistema comunal en que está pensando Patzi Paco donde la propiedad colectiva se combina con la gestión y utilización privada, en la economía comunal practicada por los comuneros de la Colonia, los recursos naturales, la tierra y los medios de trabajo son distribuidos y utilizados de forma comunal. En la Colonia no se reproducen los patrones de diferenciación ni separación entre dominios políticos, económicos, culturales, pues funciona como "un sistema único que relaciona el entorno interno y el externo" en cuya relación se apropia del entorno liberal y lo subordina a la lógica comunal volviéndose más competitivo y justo (Patzi, 2004, pp. 171-172).

La interacción entre un sistema comunal y su entorno puede llevar a indagar la fortaleza o la vulnerabilidad de aquel sistema para reproducirse, valiéndose de factores contextuales favorables (mediante el aprovechamiento de las políticas públicas) y para sobrevivir a situaciones exógenas desfavorables (recorte de presupuesto, existencia de monopolios que desplazan la producción comunal de los mercados, resistencias al desalojo) (Jara, et al., 2018). Colonia Jaime tuvo la capacidad de reproducirse en contextos hostiles y favorables al generar "un desarrollo importante de las fuerzas productivas que le permitió crear un proceso de acumulación considerable" (Suárez y Paz, 2017, p. 98) que mejoró las condiciones materiales de existencia de los comuneros a partir de una organización económica comunal donde no existe el salario, ni la propiedad privada sino una redistribución equitativa que busca satisfacer todas las necesidades individuales y colectivas en salud, vivienda, educación, consumo, producción, recreación. Queda así manifestada su propia lógica productiva comunal no subordinada al capital.

Por otra parte, Colonia Jaime "muestra otra forma de producir alimento y de vivir". Ello abre líneas conceptuales que ayudan a pensar en formas otras con "un diseño de producción y de vida que implica indagar en una teoría del valor diferente a la planteada por el capitalismo, y explorar la potencialidad de esa forma de vida para plantear otras alternativas" (Suárez y Paz, 2018, p. 7). "La teoría clásica del valor requiere necesariamente la separación del hombre y la naturaleza" como también exige de la profundización del individualismo y la competencia extrema; ello significa "la disolución del organismo económico en sus elementos, de modo que cada uno de ellos pueda encajar en la parte del sistema donde fuese más útil y eficiente" (Paz, 2017, p. 59). 
Esta perspectiva no solo modifica la forma de concebir a la teoría del valor desde la dimensión económica, sino también genera cambios profundos que se producen en los modos de pensamiento y de comprensión en ver al mundo, en la forma de vida, en los procesos políticos e institucionales, en las tecnologías, en las relaciones sociales, en las costumbres, preferencias y aspectos culturales que conforman la vida cotidiana.

\section{Referencias}

Barkin, D. y Rosas, M. (2006). ¿Es posible un modelo alterno de acumulación? Una propuesta para la Nueva Ruralidad. Polis, 13, 1-9. http://polis.revues.org/5442

Diario Panorama. (31 de agosto de 2012). El gobernador presidió el acto por el aniversario de Colonia Jaime. Diario Panorama. Recuperado de https://www.diariopanorama.com

Flores, C.; Zizumbo Villarreal, L.; Cruz, G. y Vargas, E. (2014). Economía social, comunalidad: orientación teórica para el turismo rural, como alternativa de desarrollo. Revista Mexicana de Ciencias Agrícolas, 5(9), 1645-1658. http://www.redalyc.org/articulo.oa?id=263137781010

García, Á. (2009). Forma valor y forma comunidad. Aproximación teórica-abstracta a los fundamentos civilizatorios que preceden al Ayllu Universal. Bolivia: Clacso

Giménez, C. (1991). Valdelaguna y Coatepec: permanencia y funcionalidad del régimen comunal agrario en España y México. España: Ministerio de Agricultura, Pesca y Alimentación.

Gracia, M. E. (2015). Trabajo, reciprocidad y re-producción de la vida. Experiencias colectivas deautogestión y economía solidaria en América Latina. Argentina: Miño y Dávila, ECOSUR.

Gutiérrez, M. y González, V. (2016). Desarrollo rural, política pública y agricultura familiar. Reflexiones en torno a experiencias de la Agricultura Familiar en Santiago del Estero. Argentina: Magna

Hinkelammert, F. y Mora, H. (2005). Hacia una economía para la vida. Costa Rica: Dei.

Jara, C.; Hoffman, M.; Palomo Garzón, V. (2018). Los Sistemas comunales en Santiago del Estero. Consideraciones teóricas y epistemológicas. En Paz Raúl, Cristian Jara y Ramiro Rodríguez (Comp.) Sistemas comunales, estructura agraria y explotaciones sin límites definidos: miradas alternativas, dilemas y disyuntivas. Argentina: Edunse 
Martínez, J. (2003). Comunalidad y desarrollo, México: CONACULTA.

Marx, K. (1987). Formaciones económicas precapitalistas. México: Siglo XXI.

Meillassoux, C. (1997). Mujeres, graneros y capitales, México: Siglo XXI.

Osorio, A. (2013). La comunalidad como herramienta: una metáfora espiral. Cuaderno del Sur, 18(34), 39-56. http://cuadernosdelsur.com/revistas/34-enero-junio-2013/

Patzi, F. (2004). Sistema comunal. Una propuesta alternativa al sistema liberal. Bolivia: CEA.

Paz, R. (2017). Las grietas de los agronegocios y los imperativos de la agricultura familiar: hacia una perspectiva conceptual. Revista Latinoamericana de Estudios Rurales, 2(3), 3963. Recuperado de: http://www.ceilconicet.gov.ar/ojs/index.php/revistaalasru/article/view/194/117

Paz, R. y de Dios, R. (2011). Actores sociales y espacios protegidos. Aprendizajes de experiencias rurales en el Noroeste Argentino. Argentina: Magna.

Reglamento Interno de Colonia Jaime. (1931). Revista Luz y Verdad, Año 5(56), 4-6. Recuperado de http://circularmagna.blogspot.com/

Rodríguez, R.; Paz, R.; Suarez, M. y Díaz, J. P. (2015). Construyendo mercados desde la propia finca. Tres experiencias en la agricultura familiar. Revista Agro Sur, 43(1), 3-17. DOI: https://dx.doi.org/10.4206/agrosur.2015.v43n1-02

Ruiz, E. (2012). La vigencia de la comunidad. Prácticas para navegar en la globalización desde la periferia andina. Chungara Revista de Antropología Chilena, 44 (3), 419-433. Recuperado de: https://dx.doi.org/10.4067/S0717-73562012000300005

Santos, B. (2011). Producir para vivir. Los caminos de la producción no capitalista. México: Fondo de Cultura Económica.

Suárez, M. V. (2019). Estrategias productivas y ética espiritual en la gestión de los recursos comunes en Colonia Jaime (Santiago del Estero, Argentina) (tesis doctoral). Universidad Nacional de Tucumán, San Miguel de Tucumán, Argentina. 
Suárez, M. y Paz, R. (2017). Pensando los procesos de acumulación en sistemas comunales: desarrollo de las fuerzas productivas, innovaciones tecnológicas y Estado. El caso de Colonia Jaime, Argentina. EUTOPIA, Revista de Desarrollo Económico Territorial, (11), 97-112. https://doi.org/10.17141/eutopia.11.2017.2655

Suárez, M. y Paz, R. (2018). Vivir en sistemas comunales: contención social, procesos de acumulación y una alternativa de vida. Recuperado de: http://dspace.unipampa.edu.br:8080/jspui/bitstream/riu/3882/2/Guinter\%20Tlaija \%20Leipnitz2018.pdf

Trincado, J. (15 de abril de 1935). A propósito de colonización en la Provincia de Salta. La Balanza, 11-13.

Trincado, J. (1975). Código de Amor Universal. Para el régimen de la Comuna de Amor y Ley. Tomo II. Buenos Aires: Impresos Laflor. Segunda Edición

Trincado, J. (1983) Los Cinco Amores: Ética y Sociología. Buenos Aires: Impresos Laflor. Segunda Edición.

Van der Ploeg, J. \& Marsden, T. (2008). Unfolding Webs. The Dynamics of Regional Rural Development. The Netherlands: Royal van Gorcum.

Villoro, L. (2003). De la libertad a la comunidad. Fondo de Cultura Económica: USA 